\title{
Bile reflux and intestinal metaplasia in gastric
}

\section{mucosa}

\author{
G M Sobala, H J O'Connor, E P Dewar, R F G King, A T R Axon, M F Dixon
}

\begin{abstract}
Aim: To determine associations between enterogastric bile reflux and gastric mucosal pathology.
\end{abstract}

Method: A retrospective study using fasting gastric juice bile acid measurements and antral or prestomal biopsy specimens from 350 patients, 66 of whom had previously undergone surgery that either bypassed or disrupted the pyloric sphincter.

Results: Bile reflux was positively associated with reactive gastritis and negatively with Helicobacter pylori density. After stratification for previous surgery, age, and $H$ pylori status, the histological feature most strongly associated with bile reflux was intestinal metaplasia, including all its subtypes. The prevalence of intestinal metaplasia was greatest in patients with both $H$ pylori infection and high bile acid concentrations. Bile reflux was also positively associated with the severity of glandular atrophy, chronic inflammation, lamina propria oedema and foveolar hyperplasia.

Conclusions: Bile reflux is a cause of reactive gastritis. It modifies the features of $\boldsymbol{H}$ pylori associated chronic gastritis. The changes are not confined to patients who have had surgery to their stomachs. The positive associations with atrophy and intestinal metaplasia have implications for models of gastric carcinogenesis.

(F Clin Pathol 1993;46:235-240)

These data were presented in part at the Spring Meeting of the British Society of Gastroenterology, 1991 and were then published in brief abstract form in Gut.

Centre for Digestive Diseases, The General Infirmary, Leeds, West Yorkshire, LS1 3EX

G M Sobala

H J O'Connor

E P Dewar

R F G King

A T R Axon

M F Dixon

Correspondence to:

Dr G M Sobala

Accepted for publication

2 September 1992
Enterogastric reflux of bile has long been suspected to affect the gastric mucosa, but most histological studies of this topic have been performed before the discovery of Helicobacter pylori and its major role in the aetiology of chronic gastritis. Few clear conclusions have therefore been drawn, although we previously reported an association between bile reflux and a distinctive reflux (now "reactive") gastritis, ${ }^{1}$ and associations between bile reflux and intestinal metaplasia have also been been sought only in the context of the stomach after surgery, and has largely been neglected as a factor in non-surgical patients.

The aim of this study was therefore to determine the role of bile reflux in the causation of gastric mucosal pathology, specifically controlling for the presence of $H$ pylori, in noted. ${ }^{2-4}$ Furthermore, bile reflux has usually patients both with and without previous gastric surgery. A large study population was required to disentangle the effects of bile reflux from those of $H$ pylori infection. We achieved this by performing a meta analysis on gastric biopsy results and gastric juice bile acid measurements obtained during the course of our studies over the past 10 years. In particular, we sought to examine more closely the association we have previously reported between bile reflux and reflux or reactive gastritis, to report on the relative importance of the individual components of the histological reflux score we have previously proposed, ${ }^{1}$ and to confirm and further explore the association between bile reflux and intestinal metaplasia. We also attempted to derive a histological index predicting the presence of abnormal bile reflux.

\section{Methods}

Biopsy and gastric juice bile acid results were available from 350 patients who had participated in five published studies. ${ }^{145-7}$ The 168 patients in the first three studies had been selected for their known pathology or previous gastric surgery; 60 of these had also undergone surgery that had destroyed or bypassed the pylorus. The fourth study comprised 135 consecutive patients attending an open access endoscopy clinic, excluding those with neoplastic disease. The 47 patients in the last study were attending diagnostic endoscopy lists and were selected on criteria of convenience of the timing of sample collection and of absence of previous gastric surgery. All patients had at least two biopsy specimens taken from the antrum, or in the 35 with Billroth gastrectomies, from the remnant of stomach within $5 \mathrm{~cm}$ of the stoma.

All patients gave informed consent. The studies were individually approved by the local research ethics committee.

Gastric juice was aspirated through a nasogastric tube in the first two studies, and at the time of endoscopy in the latter three. Bile acid concentration was then determined by the steroid dehydrogenase $\operatorname{method}^{8}$ in the same laboratory throughout.

The biopsy specimens were fixed in $10 \%$ formalin. After routine processing sections were taken at three levels and stained with haematoxylin and eosin. Additional sections were stained with alcian blue $(\mathrm{pH} \mathrm{2.5)}$ and periodic acid Schiff (AB/PAS) for neutral and acidic mucosubstances and by the modified Giemsa method ${ }^{9}$ for $H$ pylori. If intestinal 
metaplasia was detected in the alcian blue/PAS stained section, new sections were cut and stained with the high iron diamine/alcian blue technique to demonstrate sulphomucins. ${ }^{10}$

The sections were examined by one pathologist (MFD) who was unaware of the bile acid results and clinical condition. The following eight features were noted and graded: chronic inflammatory cell infiltration; polymorphonuclear activity (degree of neutrophil polymorph infiltration); glandular atrophy; intestinal metaplasia; foveolar hyperplasia; oedema of the lamina propria; vasodilatation or congestion; and density of $H$ pylori colonisation. Each feature was given a numerical score $0-3$ equivalent to absent (or normal), mild, moderate and severe. The first four features and $H$ pylori density were graded in a manner analogous to that recommended recently in the Sydney system for the classification of gastritis. " The other features were graded so that a "reflux score" could be calculated. The score represents the sum of the grades for foveolar hyperplasia, lamina propria oedema, and vasodilatation or congestion, less the grades for chronic inflammation and activity ${ }^{1}$ plus 6 . Thus the minimum score is 0 and the maximum is 15 . In the past we have taken scores greater than 10 to be indicative of reflux (reactive) gastritis.

Where intestinal metaplasia was found in the stained sections, the various subtypes ${ }^{12}$ were sought by using the high iron diaminobenzidine/alcian blue sections, and the extent of each subtype (I, II, or III) was also graded on a four point scale.

The bile acid results fell in a skewed distribution and were therefore grouped into six bands before analysis: $0.00-0.05 \mathrm{mmol} / 1$, $0.06-0.20 \mathrm{mmol} / \mathrm{l}, 0.21-0.50 \mathrm{mmol} / \mathrm{l}, 0.51-$ $1.00 \mathrm{mmol} / \mathrm{l}, 1.01-5.00 \mathrm{mmol} / \mathrm{l}$, and $>5.00$ $\mathrm{mmol} / \mathrm{l}$. Associations between bile acid concentrations and the histological scores for chronic inflammation, activity, atrophy, intestinal metaplasia, foveolar hyperplasia, lamina propria oedema and vascular congestion were sought using Cochran-MantelHaenszel $\chi^{2}$ tests for non-zero correlation. The analyses were controlled by stratification for the possible confounding effects of previous surgery bypassing or disrupting the pylorus, age, and the presence of $H$ pylori.
Odds ratios and their $95 \%$ confidence intervals were calculated where appropriate. Differences in bile acids between different diagnostic groups were assessed by the nonparametric two-group Wilcoxon test.

A new histological reflux index was constructed by stepwise logistic regression. The dependent dichotomous variable was the presence or absence of an abnormal gastric juice bile acid concentration of $>1.00$ $\mathrm{mmol} / \mathrm{l}$. The independent variables were the eight graded histological features, including $H$ pylori density. The $\alpha$ values for variables to enter and remain in the analysis were set at 0.05 . The analysis was terminated when the residual $\chi^{2}$ test for variables not included became non-significant.

Statistical analyses were performed using the SAS package v6.04 and EPI-INFO v5.0 on an IBM compatible personal microcomputer.

\section{Results}

Three hundred and fifty patients (212 men) were studied between 1979 and 1990. The median age was 49 years, range 18 to 88 .

The fasting gastric bile acid concentrations in each diagnostic group are shown in table 1.

Seven of $158(4 \cdot 4 \%)$ patients with normal endoscopies had bile acid concentrations greater than $1.00 \mathrm{mmol} / 1$, confirming that this value can be considered the upper limit of the normal range.

Concentrations were substantially higher in patients who had undergone surgery that either bypassed or disrupted the pyloric sphincter. Fifteen of the 43 patients with bile concentrations above $1.00 \mathrm{mmol} / 1$ and 25 of the 30 patients with concentrations between 0.50 and $1.00 \mathrm{mmol} / \mathrm{l}$ had not undergone such surgery.

\section{HISTOLOGICAL FEATURES}

Fiftytwo patients had normal gastric mucosa, of whom $3.9 \%$ had visible $H$ pylori on histological examination, 219 patients had chronic gastritis, (94.0\% $H$ pylori positive), and 79 patients had reactive gastritis $(16.5 \% \mathrm{H}$ pylori positive).

Patients with $H$ pylori were less likely to have bile acid concentrations above $1.00 \mathrm{mmol} / 1$ (26 of $129(20 \cdot 2 \%) H_{\text {pylori neg- }}$

Table 1 Bile reflux $v$ endoscopic diagnosis

\begin{tabular}{|c|c|c|c|c|c|}
\hline Diagnosis & $n=$ & Lower quartile & Median & Upper quartile & p Value \\
\hline Normal & 158 & 0.00 & 0.00 & $0 \cdot 10$ & - \\
\hline Duodenal ulcer & 51 & 0.00 & $0 \cdot 10$ & 0.30 & ns \\
\hline Gastric ulcer & 41 & 0.00 & 0.00 & 0.30 & ns \\
\hline $\begin{array}{l}\text { Duodenal and } \\
\text { gastric ulcer }\end{array}$ & 11 & 0.00 & 0.00 & 0.30 & ns \\
\hline Pernicious anaemia & 6 & 0.00 & 0.05 & $1 \cdot 10$ & ns \\
\hline Highly selective & & & & & \\
\hline $\begin{array}{l}\text { vagotomy } \\
\text { Vagotomy and }\end{array}$ & 23 & 0.00 & 0.00 & $0 \cdot 10$ & ns \\
\hline $\begin{array}{l}\text { pyloroplasty } \\
\text { Vagotomy and }\end{array}$ & 12 & 0.00 & 0.20 & 0.95 & 0.07 \\
\hline gastrojejunostomy & 13 & 0.30 & 0.50 & $3 \cdot 70$ & $<0.0001$ \\
\hline Billroth I & 19 & 0.00 & 0.40 & $2 \cdot 70$ & $<0.001$ \\
\hline Billroth II & 16 & 1.00 & 2.90 & $9 \cdot 85$ & $<0.0001$ \\
\hline
\end{tabular}

Fasting gastric juice bile acid concentrations (medians and interquartile range; $\mathrm{mmol} / \mathrm{l}$ ) in the 10 different diagnostic groups; values indicate whether results differ significantly from normal group, two tailed Wilcoxon test. 
Table 2 Bile reflux: associations with histological features

\begin{tabular}{lcll}
\hline $\begin{array}{l}\text { Histological } \\
\text { feature }\end{array}$ & $\begin{array}{l}\text { Mantel-Haenszel } \\
\chi^{2} \\
\text { statistic }(d f=1)\end{array}$ & $p$ Value & $\begin{array}{l}\text { Direction of } \\
\text { Association }\end{array}$ \\
\hline Chronic inflammation & 4.03 & $\mathrm{p}<0.05$ & Positive \\
Polymorphonuclear activity & 2.24 & $\mathrm{NS}$ & Positive \\
Glandular atrophy & 8.24 & $\mathrm{p}<0.01$ & Positive \\
Intestinal metaplasia & 17.29 & $\mathrm{p}<0.001$ & Positive \\
Foveolar hyperplasia & 4.56 & $\mathrm{p}<0.05$ & Positive \\
Lamina propria oedema & 7.78 & $\mathrm{p}<0.01$ & \\
Vascular congestion & 0.73 & $\mathrm{NS}$ & \\
\hline
\end{tabular}

Cochran-Mantel-Haenszel statistics for non-zero correlations between four-point histological scores and bile acid concentrations, analyses stratified for presence of previous surgery bypassing or disrupting the pyloric sphincter, age and $H$ pylori status.

ative $v 17$ of $221(7 \cdot 7 \%) H$ pylori positive; $\mathrm{p}<$ $\left.0.001 \chi^{2}\right)$.

In patients with $H$ pylori infection, there was a negative association between the histological density of infection and bile acid concentrations (Cochran-Mantel-Haenszel test for non-zero correlation, stratified for previous surgery and age; $\chi^{2}=5.45 ; p<0.05$ ).

Associations between the seven graded histological features and bile acid concentrations, stratified for previous surgery, age, and $H$ pylori status, are shown in table 2 . There were significant positive associations with the degrees of foveolar hyperplasia, chronic inflammation, lamina propria oedema and glandular atrophy, and a highly significant positive association with the degree of intestinal metaplasia.

INTESTINAL METAPLASIA

Sixtythree of the 82 patients with intestinal metaplasia had chronic gastritis and the rest

Table 3 Bile reflux $v$ intestinal metaplasia and reactive gastritis

\begin{tabular}{|c|c|c|c|c|c|}
\hline \multirow{2}{*}{$\begin{array}{l}\text { Bile acid } \\
\text { concentrations } \\
\text { (mmolll) }\end{array}$} & \multirow[b]{2}{*}{$n=$} & \multicolumn{3}{|c|}{$\begin{array}{l}\text { (a) \% Prevalence of } \\
\text { intestinal metaplasia }\end{array}$} & \multirow{2}{*}{$\begin{array}{l}\text { (b) Odds ratio ( } 95 \% \text { CI) } \\
\text { for reactive gastritis }\end{array}$} \\
\hline & & Focal & Moderate & Extensive & \\
\hline $\begin{array}{l}0.00-0.05 \\
0.06-0.20 \\
0.21-0.50 \\
0.51-1.00 \\
1.01-5.00 \\
>5.01\end{array}$ & $\begin{array}{r}181 \\
58 \\
38 \\
30 \\
26 \\
17\end{array}$ & $\begin{array}{l}13 \cdot 8 \\
12 \cdot 1 \\
13 \cdot 2 \\
23 \cdot 3 \\
30 \cdot 8 \\
23 \cdot 5\end{array}$ & $\begin{array}{r}3 \cdot 3 \\
8 \cdot 6 \\
2 \cdot 6 \\
6 \cdot 7 \\
11 \cdot 5 \\
11 \cdot 8\end{array}$ & $\begin{array}{r}1 \cdot 1 \\
1 \cdot 7 \\
0 \cdot 0 \\
3 \cdot 3 \\
3 \cdot 8 \\
11 \cdot 8\end{array}$ & $\begin{array}{c}1.00 \text { (baseline) } \\
0.96(0 \cdot 42-2 \cdot 19) \\
2.04(0.89-4.67) \\
0.78(0 \cdot 25-2 \cdot 47) \\
4.36(1.53-12 \cdot 41) \\
13 \cdot 19(3 \cdot 70-47.08)\end{array}$ \\
\hline
\end{tabular}

(a) Percentage prevalences of each grade of intestinal metaplasia (focal, moderate, extensive) according to bile acid concentration.

(b) Mantel-Haenszel odds ratios and $95 \%$ confidence limits for the presence of reactive gastritis according to bile acid concentration, stratified for previous surgery bypassing or disrupting the pylorus. $\chi^{2}$ for trend $=16.0 ; p<0.001$.

Table 4 Intestinal metaplasia, $H$ pylori, and bile reflux

\begin{tabular}{llllll}
\hline $\begin{array}{l}\text { H pylori } \\
\text { present? }\end{array}$ & $\begin{array}{l}\text { Bile reflux } \\
>1 \text { mmolll? }\end{array}$ & $n=$ & Focal & Moderate & Extensive \\
\hline No & No & 103 & $5 \cdot 8$ & 1.9 & 1.9 \\
Yes & No & 204 & 18.6 & 5.9 & 1.0 \\
No & Yes & 26 & $30 \cdot 8$ & 3.9 & 7.7 \\
Yes & Yes & 17 & 23.5 & 23.5 & 5.9 \\
\hline
\end{tabular}

Prevalence of each grade of intestinal metaplasia (focal, moderate, extensive) according to presence of $H$ pylori and of abnormal bile reflux.

Table 5 Logistic regression: predictors of bile reflux

\begin{tabular}{llll}
\hline Variable & $p$ Value & Odds ratio & $(95 \%$ CI) \\
\hline Lamina propria oedema & $\mathrm{p}<0.0001$ & 4.03 & $(2.36-6.86)$ \\
H pylori density & $\mathrm{p}<0.0001$ & 0.30 & $(0.17-0.53)$ \\
Chronic inflammation & $\mathrm{p}<0.001$ & 2.33 & $(1.44-3.76)$ \\
Intestinal metaplasia & $\mathrm{p}<0.05$ & 1.81 & $(1.14-2.88)$ \\
\hline
\end{tabular}

Results of stepwise logistic regression to predict a fasting gastric juice bile acid concentration of $>1.00 \mathrm{mmol} / \mathrm{l}$. The odds ratios reflect the change in the likelihood of a high bile concentration associated with an single increment in the score of each histological variable. had reactive gastritis. The relation between bile acid concentrations and intestinal metaplasia is shown in table 3.

The prevalence of intestinal metaplasia was greatest in patients with both $H$ pylori infection and a bile concentration of $>1.00$ $\mathrm{mmol} / \mathrm{l}$ (table 4).

Fifty patients had type I intestinal metaplasia, 53 had type II, and five had type III: 24 patients had more than one type. Focal intestinal metaplasia was usually of type II while extensive intestinal metaplasia was largely of type I. Each individual type of intestinal metaplasia occurred more often in patients with bile acid concentrations above $1.00 \mathrm{mmol} / \mathrm{l}$ than in those with lower bile acid concentrations (type $I$, odds ratio $=3 \cdot 2,95 \%$ confidence interval $=1.4-7.0, \mathrm{p}<0.01$; type II, odds ratio $=3.3(1.5-7 \cdot 2), \mathrm{p}<0.01$; type III, odds ratio $=11.4(1.3-139), \mathrm{p}<0.05)$. Extensive type I intestinal metaplasia in a patient with bile reflux but without previous gastric surgery is shown in fig 1 .

\section{BILE REFLUX AND REACTIVE GASTRITIS}

Reactive gastritis was diagnosed when the previously defined "reflux score" was greater than 10. Such a diagnosis was more likely when bile acid concentrations were high (table 3). The sensitivity of a diagnosis of reactive gastritis for a gastric juice bile acid concentration of $>1.00 \mathrm{mmol} / \mathrm{l}$ was $60 \%$, and the specificity $83 \%$.

\section{NEW HISTOLOGICAL BILE REFLUX INDEX}

The results of the logistic regression are shown in table 5. A new index predictive of bile reflux was constructed from the regression coefficients: (new index) $=(7 \times$ lamina propria oedema $)+(3 \times$ intestinal metaplasia $)+(4 \times$ chronic inflammation $)-(6 \times$ $H$ pylor). A cutoff above 14 gave the highest combined sensitivity $(70 \%)$ and specificity $(85 \%)$ for bile reflux of $>1.00 \mathrm{mmol} / \mathrm{l}$.

\section{Discussion}

The limitations of this study must be considered before any conclusions are drawn. There were sampling problems. Although analysis of bile acid concentration in fasting gastric juice correlates well with other objective techniques of determining bile reflux, a single measurement does not indicate the duration and may misrepresent the degree of bile reflux experienced by an individual. ${ }^{13}$ Histological changes are often patchy and two mucosal biopsy specimens are not always representative. Therefore such a study will underestimate the strength of any true associations between bile reflux and gastric mucosal histology. Any apparent associations may have been due to exposure to substances other than bile in the enterogastric refluxate. Finally, this study is not an independent confirmation of our previously reported findings as there was overlap of subjects. Nevertheless, the results are important as this series is much larger than any published before in this field, with greater statistical power. It is also the 
Figure 1 Type I intestinal metaplasia in a patient with fasting bile acid concentration of 1.92 mmoll and an unoperated stomach.



first to grade biopsy specimens in a manner analogous to that of the new Sydney classification of gastritis, ${ }^{11}$ although the two corpus biopsy specimens required for a full classification were not available.

Several studies have attempted to correlate bile reflux with chronic inflammatory infiltration in the gastric mucosa, usually unsuccessfully. ${ }^{14} 15$ It is now known that chronic gastritis is aetiologically linked to infection with $H$ pylori $^{16}$ and that the intensity of inflammation is related to the histological



Figure 2 Antral biopsy specimen from a patient with an unoperated stomach and a fasting bile acid concentration of $8.95 \mathrm{mmol} / \mathrm{l}$, showing intestinal metaplasia and moderate chronic inflammatory cell infiltration. Scanty Helicobacters were present. Diagnosed as reactive gastritis (reflux score $=11$ ), this probably represents a transitional case. density of infection. ${ }^{17} 18$ This correlation is strong. Previous studies have failed to take it into account and any effect of bile will have been masked. Controlling for the presence of $H$ pylori infection, we did find a weak correlation between bile reflux and the density of the chronic inflammatory infiltrate. We also observed a negative association between bile and $H$ pylori density and so we conclude that scantiness of $H$ pylori with severe chronic inflammation should alert the observer to the possibility of bile reflux. The logistic regression confirmed that chronic inflammation positively, and density of $H$ pylori infection negatively, predict raised gastric juice bile concentrations. These findings lend some support to our previously stated hypothesis that bile reflux causes a gradual elimination of $H$ pylori and subsequent resolution of the chronic inflammatory response, ultimately giving rise to the distinctive reactive gastritis picture. This phenomenon has so far only been observed in the stomach after surgery. ${ }^{19}$ During this process intermediate stages are represented by few or no Helicobacters with persistent chronic inflammatory cell infiltration (fig 2).

We also found that many patients with reactive gastritis had little or no demonstrable bile reflux. This highlights the likelihood that other chemical irritants such as non-steroidal anti-inflammatory drugs and possibly alcohol cause the same histological picture. ${ }^{20-22} \mathrm{We}$ did not have detailed drug or alcohol history on sufficient patients to control for these factors.

This study casts some doubts on the validity of the construction of the original "reflux score". No association was found between vascular congestion and bile reflux, and the association with chronic inflammation was positive rather than negative. However, the negative emphases given to activity and chronic inflammation in the original score usefully served to bias it against $H$ pylori associated chronic gastritis: few patients with Helicobacter associated chronic gastritis also have reflux scores greater than 10 . Thus reactive gastritis, so defined, is a distinct histological entity ("foveolar hyperplasia, oedema and congestion without cellular inflammation").

The new index derived by logistic regression differs in concept from the original reflux score. This is an attempt to predict the presence of bile reflux, rather than to define a new histological entity. Lamina propria oedema, intestinal metaplasia, and a disparity between $H$ pylori density and the degree of chronic inflammation were found independently to predict bile reflux.

Associations between bile reflux and intestinal metaplasia have been reported before but usually only following gastric surgery. ${ }^{2}{ }^{23}$ We suggest that bile reflux is also a factor in the production of intestinal metaplasia in the unoperated stomach. The mechanism of this association is debatable. The currently dominant hypothesis is that intestinal metaplasia is a precancerous condition, occurring as a result of exposure to 
Figure 3 Diagram summarising hypothesis of the causation of intestinal metaplasia.
Duodenogastric bile reflux $\downarrow$

Mucosal injury, erosions

$\downarrow$

Regeneration

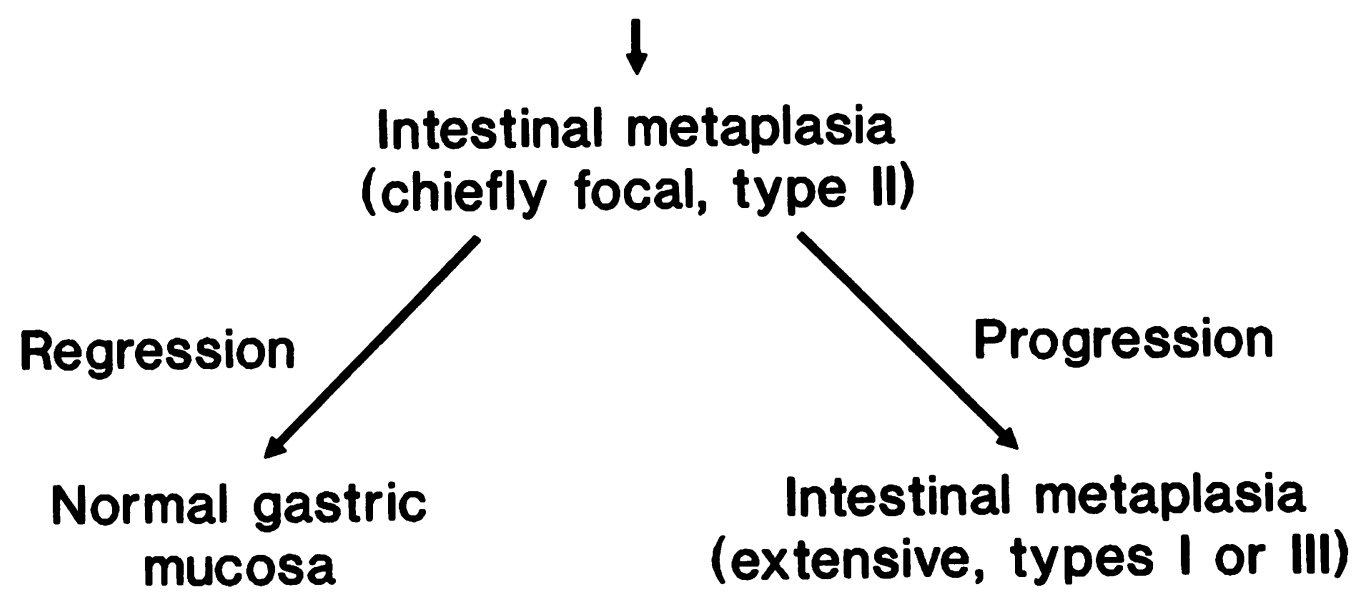

mutagens in hypochlorhydric gastric juice. ${ }^{24}$ It can certainly be argued that mutagens could be contained in or formed from the enterogastric refluxate. However, we have previously failed to find raised nitrosocompound concentrations in association with intestinal metaplasia or bile reflux. ${ }^{4}$ An alternative hypothesis is that intestinal metaplasia arises from divergent differentiation in regenerating epithelium following erosion or ulceration. There is some evidence to support this view. Bile in the presence of acid causes erosions in experimental animals, ${ }^{25}$ although this has not been shown in humans. Intestinal metaplasia seems to originate during the regenerative process in the healing of gastric erosions, ${ }^{26}$ is common in the regenerative epithelium of gastric ulcers, ${ }^{27}$ and usually regresses with time. ${ }^{28}$ When the injury is repetitive and on a background of $H$ pylori associated chronic gastritis, however, we suggest that intestinal metaplasia becomes more extensive and permanent. This hypothesis is summarised in fig 3 and is consistent with the view that intestinal metaplasia is a defence response aimed at protecting the gastric mucosa against repeated bile damage in the same way that gastric metaplasia develops in the duodenum when subjected to a high acid load, ${ }^{29}$ and gastric type epithelium appears in the lower oesophagus in response to acid reflux. This hypothesis of the genesis of intestinal metaplasia differs from that proposed by Correa. It merits further study.
1 Dixon MF, O'Connor HJ, Axon ATR, King RFJG, Johnston D. Reflux gastritis: distinct histopathological entity? I Clin Pathol 1986;39:524-30.

2 Houghton PWJ, Mortensen NJMcC, Thomas WEG, Cooper MJ, Morgan AP, Burton P. Intragastric bile acids and histological changes in gastric mucosa. $\mathrm{Br} \mathcal{F}$ Surg 1986;73:354-6.

3 Su WW, Zhao DH, Huang CX. Clinical research on the relation of chronic gastritis and intragastric bile acids. relation of chronic gastritis and intragastric

4 Sobala GM, Pignatelli B, Schorah CJ, et al. Simultaneous determination of ascorbic acid, nitrite, total nitrosocompounds and bile acids in fasting gastric juice, and gastric mucosal histology: implications for gastric carcinogenesis. Cárcinogenesis 1991;12:193-8.

5 Dewar EP, Dixon MF, Johnston D. Bile reflux and degree of gastritis in patients with gastric ulcer: before and after operation. $\mathcal{f}$ Surg Res 1984;37:277-84.

6 Dewar EP, Dixon MF, Johnston D. Bile reflux and degree of gastritis after highly selective vagotomy, and partial gastrectomy for duodenal ulcer. World $\mathcal{f}$ Surg 1983;7: 743-50.

7 Sobala GM, Dixon MF, Axon ATR. Symptomatology of Helicobacter pylori-associated dyspepsia. Eur $\mathfrak{f}$ of Helicobacter pylori-associated

8 Dewar EP, King RFGJ. Johnston D. Bile acid and lysolecithin concentrations in the stomach of patients with gastric ulcer: before operation and after treatment by highly selective vagotomy, Billroth I partial gastrectomy and truncal vagotomy and pyloroplasty. Br $\mathcal{f}$ Surg 1983;70:401-5.

9 Gray SF, Wyatt JI, Rathbone BJ. Simplified techniques for identifying Campylobacter pyloridis. ₹ Clin Pathol 1986;39:1279-80.

10 Cook HC. Carbohydrates. In: Bancroft JD, Stevens A, eds. Theory and practice of histological techniques. 2nd edn.

11 Misiewicz JJ, Tytgat GNJ, Goodwin CS, et al. The Sydney system: a new classification of gastritis. $\boldsymbol{f}$ Hepatol system: a new classificatio

12 Filipe MI, Jass JR. Intestinal metaplasia subtypes and cancer risk. In: Current problems in tumour pathology: gastric carcinoma. Edinburgh: Churchill Livingstone, 1986: 87-115.

13 Houghton PWJ, Mortensen NJMcC, Thomas WEG, Cooper MJ, Morgan AP, Davies ER. Intragastric bile acids and scintigraphy in the assessment of duodenogastric reflux. Br $\mathcal{F}$ Surg 1986;73:292-4.

14 Bechi P, Amorosi A, Mazzanti R, Romagnoli P, Tonelli L. Gastric histology and fasting bile reflux after partial gastrectomy. Gastroenterology 1987;93:335-43. 
15 Watt PCH, Sloan JM, Spencer A, Kennedy TL. Histology of the postoperative stomach before and after diversion of bile. $\mathrm{Br}$ Med $\mathcal{F} 1983 ; 287: 1410-12$.

16 Morris A, Nicholson G. Ingestion of Campylobacter pyloridis causes gastritis and raised fasting gastric $\mathrm{pH}$. Am $\mathcal{Y}$ Gastroenterol 1987;82:192-9.

17 Steininger $H$, Schneider U, Bartz $K$, Simmier B. Campylobacter pylori und gastritis-Beseidelungsdichte Campylobacter pylori und gastritis-Beseidelungsdichte phometrische Untersuchung. Leber Magen Darm 1989; 19:70-8.

18 Stolte M, Eidt S, Ohnsmann A. Differences in Helicobacter pylori associated gastritis in the antrum and body of the stomach. Z Gastroenterol 1990;28:229-33.

19 O'Connor HJ, Dixon MF, Wyatt JI, et al. Effect of duodenal ulcer surgery and enterogastric reflux on Campylobacter pyloridis. Lancet 1986;ii:1178-81.

20 Sobala GM, King RFG, Axon ATR, Dixon MF. Reflux gastritis in the intact stomach. $f$ Clin Pathol 1990;43: 303-6.

21 Laine L, Marin-Sorensen M, Weinstein WM. The histology of gastric erosions in patients taking non-steroidal anti-inflammatory drugs (NSAID's): a prospective study. Gastroenterology 1988;94:A247.

22 Laine L, Weinstein WM. Histology of alcoholic haemorrhagic "gastritis": a prospective evaluation. Gastroenterology 1988;94:1254-62.

23 Ishii T. Experimental study on the atrophic gastritis especially on the cause of the postoperative gastritis and on the histogenesis of experimentally induced atrophic gastritis. Ipn I Gastroenterol 1966;63:1323-37.

24 Correa P. A human model of gastric carcinogenesis. Cancer Res 1988;48:3554-65.

25 Smith GM. An experimental study of the relation of bile to ulceration of the mucous membrane of the stomach. to ulceration of the mucous
$\mathfrak{f}$ Med Res 1914;30:147-84.

26 Mukawa $\mathrm{K}$, Nakamura T, Nakano G, Nagamachi $Y$ Histopathogenesis of intestinal metaplasia: minute lesions of intestinal metaplasia in ulcerated stomachs. $\mathcal{f}$ lesions of intestinal metaplas

27 Oohara T, Tohma H, Aono G, Ukawa S, Kondo Y. Intestinal metaplasia of the regenerative epithelia in 549 gastric ulcers. Hum Pathol 1983;14:1066-71.

28 Silva S, Filipe MI, Pinho A. Variants of intestinal metaplasia in the evolution of chronic atrophic gastritis and gastric ulcer. A follow-up study, Gut 1990;31:1097-104. 29 Wyatt JI, Rathbone BJ, Sobala GM, et al. Gastric epithelium in the duodenum: its association with Helicobacter pylori and inflammation. $f$ Clin Pathol 1991;43:981-6. 